\title{
Improved Method of Neutron Activation Analysis for Microleakage Studies
}

\author{
J. M. MEYER,* J. B. DENNISON, and R. G. CRAIG
}

School of Dentistry, University of Michigan, Ann Arbor, Michigan 48104, USA

Improvements in the neutron activation technique for studying microleakage were achieved by selecting more suitable tracers and by using a more accurate method for evaluation of the activation data. Dysprosium was the most suitable tracer; it provided the least variation in the results and allowed the fastest activation and counting procedure.

A quantitative method for studying microleakage in vivo and in vitro has been presented by Going, Myers, and Prussin ${ }^{1}$ and Going2; they used neutron activation of manganese 55 . Teeth restored in vitro or in vivo were soaked in an aqueous solution of a nonradioactive $M n$ salt by use of a test tube and a latex isolator, respectively. After immersion, the teeth restored in vivo were extracted. The whole teeth were placed in the core of a nuclear reactor; the $55 \mathrm{Mn}$ was activated to $56 \mathrm{Mn}$ and the $\gamma$-ray emission of the ${ }^{56} \mathrm{Mn}$ formed during the irradiation was measured; the number of radioactive counts was proportional to the uptake of $\mathrm{Mn}$ per tooth.

This method was used later by Dennison,3 but he did not succeed in showing statistically significant differences between the Mn uptake values obtained from whole teeth restored with composite resins and those restored with a silicate cement. The main difficulty was the variability of the results; this was caused partially by the presence of $\mathrm{Mn}$ in either the tooth itself or in the restorative material.

The purpose of this study was to improve the efficiency of the activation method by

This paper was presented, in part, at the 51 st General Session of the IADR in Washington, DC, April 1973.

Received for publication June 22, 1973.

- Present address: Ecole de Medecine Dentaire, 30, rue Lombard, 1211 Geneva 4, Switz. selecting a more specific tracer, by shortening the activation and counting procedures, and by designing a more accurate means for analysis of the data.

\section{Materials and Methods}

Materials.-Selection of a tracer involves consideration of the following characteristics: nuclear reaction, half-life, activation cross section, natural abundance of the stable isotope, and its absence in tooth and restorative materials. Such data for Mn, vanadium $(\mathrm{Vn})$, indium (In), and dysprosium (Dy) are listed in Table 1. The latter three were selected on the basis of the aforementioned criteria. ${ }^{4-6}$ The relative activity value was obtained from a relation including neutron flux, cross section, natural abundance, and atomic weight of the element.

By developing the same relation for factors such as activation, decay, and counting times, it was possible to predict the total activity for a mass unit and for all the possible activation and counting conditions. Such calculations were performed for the tracers selected and for the most sensitive of the elements present in teeth or restorative materials. With a constant activation time of one minute and a counting time of 40 seconds, the values obtained for decay times such as $1,3,10$, and 30 minutes are reported in Figure 1. It is apparent that Dy has the shortest half-life and the greatest activity between one and eight minutes of decay. The decrease in activity for the sensitive elements present in teeth does not change notably during the same period. To maintain the best sensitivity for the proposed tracers the following conditions were chosen for all further experiments: activation, one minute; decay, three minutes; and counting, 40 seconds, plus automatic correction for the 
TABLE 1

Properties of the Selected Tracers

\begin{tabular}{|c|c|c|c|c|c|c|}
\hline & ${ }^{55} \mathrm{Mn}$ & ${ }^{51} \mathrm{~V}$ & ${ }^{2105} \mathbf{I n}$ & ${ }^{115}$ In & ${ }^{104} \mathrm{Dy}$ & ${ }^{181} \mathrm{Dy}$ \\
\hline Natural abundance (\%) & 100.0 & 99.56 & 95.7 & 95.7 & 28.18 & 28.18 \\
\hline Nuclear reaction & $(n, \gamma)$ & $(n, \gamma)$ & $(n, \gamma)$ & $(n, \gamma)$ & $(n, \gamma)$ & $(n, \gamma)$ \\
\hline Radioactive isotope & ${ }^{58} \mathrm{Mn}$ & $52 \mathrm{~V}$ & ${ }^{110 m}$ In & ${ }^{116 m}$ In & ${ }^{165} \mathrm{Dy}$ & ${ }^{165 \mathrm{~m}}$ Dy \\
\hline Energy (kev) & 850 & 1,430 & 1,085 & 1,270 & 95 & 108 \\
\hline Half-life (min) & 155.0 & 3.77 & 54.0 & 54.0 & 137.0 & 1.25 \\
\hline \multicolumn{7}{|l|}{ Activation cross section } \\
\hline (barns) & 13.3 & 4.5 & 155.0 & 155.0 & 800.0 & $2,000.0$ \\
\hline \multicolumn{7}{|l|}{ Relative activity* (disinte- } \\
\hline grations $/ \sec \times 10^{10}$ ) & 7.26 & 2.64 & 20.93 & 29.07 & & 100.9 \\
\hline
\end{tabular}

- Calculated for a flux of $5 \times 10^{12} \mathrm{n} / \mathrm{cm}^{2} /$ second and a theoretical counter with equal efficiency between 95 and $1,430 \mathrm{kev}$.

dead time of the counter. Standards were tested first and the tracers then were used on teeth restored with the same composite resin to compare practical efficiency.

The selection of valid counts in the energy range of interest in the $\gamma$-ray emission spectrum was based on the comparison of the actual counts for a given channel with a critical level, 7 which was obtained from the standard deviation of a prepared blank. This blank was constructed by weighting the counts of separate tooth and material blanks according to the actual weights of the tooth and filling material in the restored tooth to be tested, and adding them channel by channel. This procedure enables a computer to select automatically the first and the last significant channel of a given $\gamma$ peak in the complex spectrum obtained from the restored tooth, and permits the determination of items such as background and net area of the peak.

EXPERIMENTAL PRocedURE.-Two aqueous solutions were prepared for each tracer: a soaking solution ( $(\mathrm{gm} / 100 \mathrm{ml})$ and a standard solution $(100 \mu \mathrm{g} / \mathrm{ml})$. The following salts were selected: $\mathrm{MnSO}_{4} \cdot \mathrm{H}_{2} \mathrm{O},{ }^{a} \mathrm{~V}_{2} \mathrm{O}_{5},{ }^{a}$ In $\left(\mathrm{NO}_{3}\right)_{3} \cdot 3 \mathrm{H}_{2} \mathrm{O}, \mathrm{b}$ and $\mathrm{Dy}\left(\mathrm{NO}_{3}\right)_{3} \cdot 5 \mathrm{H}_{2} \mathrm{O}$.c All salts except $\mathrm{V}_{2} \mathrm{O}_{5}$, were readily soluble in water. According to the procedure described by Willard and Diehl, 8 small portions of $\mathrm{V}_{2} \mathrm{O}_{5}$ were first treated with ammonium hydroxide, then transformed into vanadic acid by nitric acid, and finally reduced to vanadyl ions through oxalic acid treatment. The solution then was neutralized and adjusted to the desired concentration.

- J. T. Baker Chemical Co., Philadelphia, NJ.

b E. H. Sargent and Co., Detroit, Mich.

c Pfaltz and Bauer, New York, NY.
Three types of filling materials were used: a composite resin $(A), \mathrm{d}$ an unfilled resin $(S), e$ and a silicate cement $(M){ }^{\perp}$ Filling materials were condensed into a small split mold to form disks, which were used as material blanks.

Freshly extracted, sound premolars and molars were used. Round Class $\mathrm{V}$ cavities were prepared on the buccal surfaces of these teeth by use of a conventional technique after storage for at least 24 hours in distilled water. Each restoration had a relatively constant exposed surface area of 12.5 $\mathrm{mm}^{2}$ and was placed to minimal depth within the dentin. After preparation and an additional hour of storage in water, the tooth was dried with compressed air for 30 seconds and weighed. The tooth was filled and after the finishing was accomplished, it was stored another hour in distilled water. The restored tooth then was dried again with compressed air and weighed. This procedure allowed good repeatability of the weighings. All tooth surfaces then were sealed with utility waxg applied with a hot spatula to within $0.5 \mathrm{~mm}$ of the margin in the restored region. Special care was taken to seal the apex region and the enamel surface adjacent to the margins so that fluid exchange could take place only along the margins of the restorations. Waxing all the surfaces ensured a close contact between wax and tooth surface, and finally an additional layer of wax was applied. In Figure 2 the standard cavity preparation used throughout the study is shown, with a similar NJ.

d Adaptic, Johnson \& Johnson Co., New Brunswick, Sevriton, Amalgamated Dental Trade Distribution, Ltd., London, Eng.

I MQ, S. S. White Co., Philadelphia, Pa.

g Kerr Manufacturing Company, Romulus, Mich. 


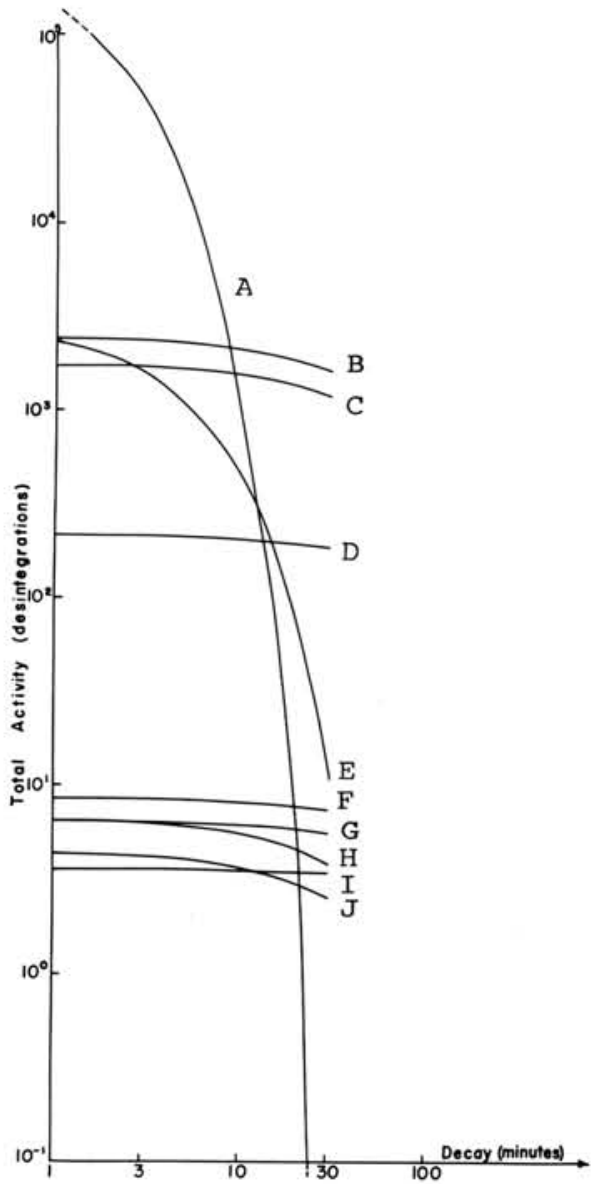

Fic 1.-Theoretical total activities calculated for various decay times for a mass unit activated for one minute and counted for 40 seconds. $A$, ${ }^{105 \mathrm{~m}}$ Dy $(108 \mathrm{kev}) ; B,{ }^{116 \mathrm{~m}}$ In $(1,270 \mathrm{kev}) ; C,{ }^{116 \mathrm{~m}} \mathrm{In}$ $(1,085 \mathrm{kev}) ; D,{ }^{50} \mathrm{Mn}(850 \mathrm{kev}) ; E,{ }^{52} \mathrm{~V}(1,430 \mathrm{kev})$; $F,{ }^{28} \mathrm{Al}(1,780 \mathrm{kev}) ; G,{ }^{31} \mathrm{P}\left(n_{\alpha}\right),{ }^{28} \mathrm{Al}$ (1,780 kev); $H,{ }^{38} \mathrm{Cl}(2,160 \mathrm{kev}) ; I,{ }^{24} \mathrm{Na}(1,370 \mathrm{kev}) ; J,{ }^{38} \mathrm{Cl}$ $(1,640 \mathrm{kev})$. Possible tracers (Dy, In, V, and Mn) are compared with most sensitive elements present in teeth or restorative materials (Al, P, $\mathrm{Cl}$, and $\mathrm{Na}$ ).

tooth that was waxed and sealed for soaking in the tracer solution. The waxed specimens were soaked for an hour in one of the concentrated salt solutions, then quickly rinsed in distilled water and wiped off with absorbing paper, except for the restored surface. An oversized piece of green wax was sealed over the restoration to prevent further seepage of the entrapped tracer solution, especially during the activation procedure.

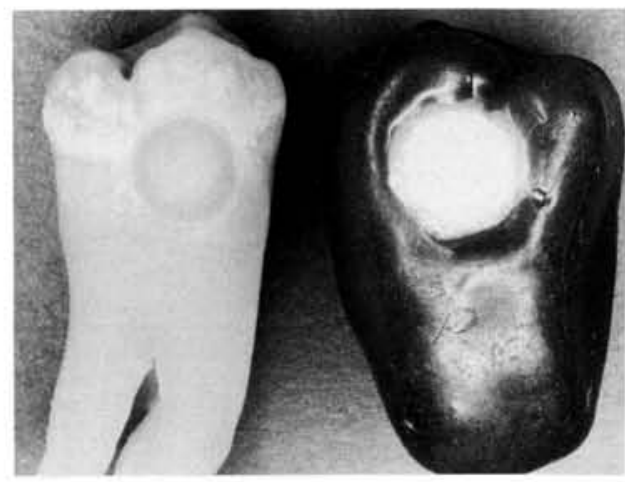

FIG 2.-Prepared cavity on buccal surface of molar (left) is compared with a similar cavity fully restored and waxed for soaking in tracer solution (right).

Small polyethylene tubes containing $1 \mathrm{ml}$ of the standard solution (100 $\mu \mathrm{g}$ of tracer) were used as a tracer standard. A typical neutron activation experiment included the following steps: After soaking and final waxing, a restored tooth was wrapped in polyethylene, placed in a polyethylene container, and transferred through an air tube system to the core of a nuclear reactor, ${ }^{\text {h }}$ transfer time was about four seconds. After one minute of exposure to a flux of $2 \times 10^{13}$ neutrons $/ \mathrm{cm}^{2} /$ second, the specimen was allowed to decay for three minutes before counting. The counting system included a lithium-drifted germanium solid-state detector ${ }^{1}$ (high-energy detector) or a germanium low-energy photon spectrometer ${ }^{j}$ (low-energy detector), and a 4096 channel analyzer.k Specimens were counted for 40 seconds, plus the automatic correction for the dead time of the counter. Typical total counting times ranged between 50 and 75 seconds for the high-energy detector, and between 45 and 100 seconds for the low-energy type. The tracer standard, the tooth blank, and the filling material blank then were successively irradiated according to the same procedure.

Raw data from the $\gamma$-spectrum analyzer and the experiment were submitted to a computer for analysis. A program was specifically designed for selection of the first

h Phoenix Memorial Laboratory, University of Michigan, Ann Arbor, Mich.

1 LGCU 55 SD Series 702, Princeton Gamma Tech, Princeton, NJ.

1 Model 8113-16350, Ortec Inc., Oak Ridge, Tenn.

k 4096, Nuclear Data Inc., Palatine, IIl. 
and last significant channels of the $\gamma$ peak used to determine the peak surface and that of the background below it. This program was designed so that only the highly significant peaks were selected; at least two successive channels had to have significant counts before one channel could be selected for one of the limits of the peak surface. At the same time, the peak-to-background ratio was computed, and the peak surface was corrected for decay by use of the following equations of Haerdi, Martin, and Monnier ${ }^{9}$ :

for $52 \mathrm{~V}$ and $165 \mathrm{mDy}$

$$
N_{\mathrm{o}}=\frac{\mathrm{N} \cdot \mathrm{O} \cdot 693 e^{0.693 t^{\prime} / T}}{T\left[1-e^{-0.693 t_{c}^{\prime} / T}\right]}
$$

and for ${ }^{56 \mathrm{Mn}}$ and $116 \mathrm{mIn}$

$$
N_{\mathrm{o}}=\frac{N}{e^{-0.693 t^{\prime} / T}},
$$

where $N_{o}$ is the number of counts at the end of irradiation (decay time $=0) ; N$ is the number of counts at the end of counting; $t^{\prime}$ is decay time; $t^{\prime}{ }_{c}$ is counting time; and $T$ is half-life.

Such corrections were necessary because of the important variations in the total counting time, which resulted from variations of tracer uptake and tooth weights, particularly for the short-lived isotopes such as $52 \mathrm{~V}$ and $165 \mathrm{mDy}$. The tracer uptake per tooth was finally determined by comparison with the tracer standard. Tracer uptake values calculated in this way then were compared for the different types of restorative materials by use of analysis of variance. When significant differences were found, Duncan's new multiple range test was used to rank them. Correlations of the tracer uptake with factors such as the weight of the tooth or the weight of the filling material were studied and tests for linearity and regression were performed.

\section{Results}

All of the proposed tracers were studied to determine their relative efficiency under the particular conditions of the experiment. Table 2 lists the peak area, peak-to-background ratio, and corrected peak area for the various tracers and $\gamma$ peaks; the results for the two types of detectors in the case of Dy are presented for comparison. The smaller corrected peak area obtained for $165 \mathrm{mDy}$ on the low-energy detector resulted from a much lower access time of the counter, or an in-

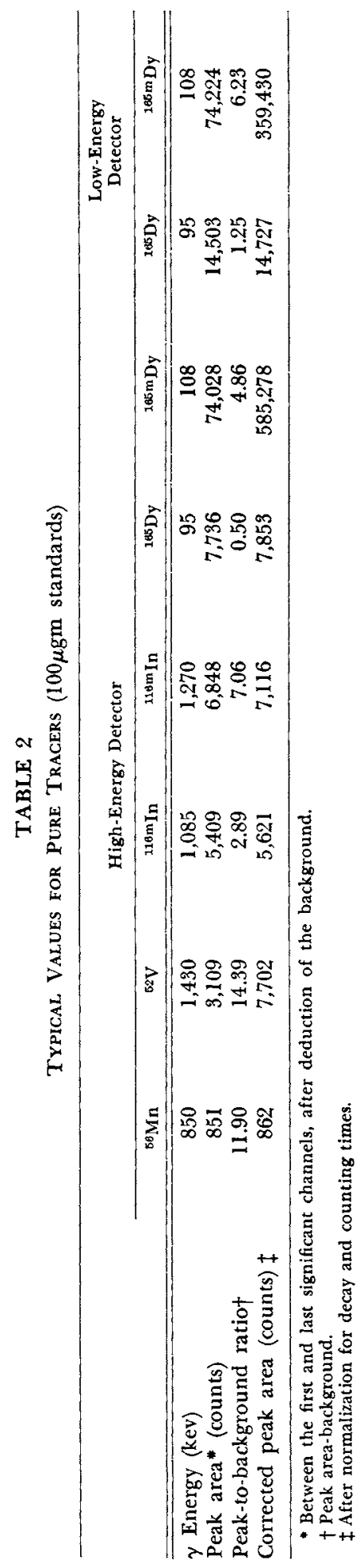


creased total counting time as a result of the greater decay of this short-lived isotope during the counting. Despite the decrease, a pronounced increase in the peak-to-background ratio was observed.

The efficiency of these tracers in microleakage tests was compared with teeth restored with the same composite resin $(A)$. Table 3 shows the number of tests in which significant peaks were detected by the automatic analysis, the mean of the peak-to-background ratios for a given tracer or $\gamma$ peak, the mean tracer uptake, the coefficient of variation within a group (when possible), and the classification of the mean tracer uptakes, based on an analysis of variance followed by a Duncan's new multiple range test modified for unequal replications. ${ }^{10}$

According to the number of successful tests, the peak-to-background ratio, and the coefficient of variation (Tables 2, 3), the $\gamma$ ray at $108 \mathrm{kev}$ for ${ }^{165} \mathrm{mDy}$ was chosen as the most suitable for microleakage studies.

An additional experiment was performed to demonstrate the capability of the method for distinguishing between marginal leakages occurring with three types of restorative materials. Table 4 shows the results obtained with an unfilled resin $(S)$ and a silicate cement $(M)$ and those already obtained with a composite resin (Table 3). The three materials were inserted into unlined cavities and there was no significant difference between the mean values obtained. It also is evident in Table 4 that there was little gained by placing a liner ${ }^{m}$ between the material and the dentin walls, except possibly to produce a greater variation in the mean values. When extreme conditions of temperautre cycling were introduced, the mean uptake values rose decidedly, but so did the variation among sample means. Student's $t$ test was used to determine a difference between mean uptake values for the composite and silicate restorations, unlined or during temperature cycling. The temperature cycling was carried out at 30-second intervals for one hour, between 10 and $60 \mathrm{C}$.

Increasing the proportion of significant counts over the background led simultaneously to a decrease in the variability of the results, because the automatic selection

\footnotetext{
NJ.

I Adaptic*, Johnson \& Johnson Co., New Brunswick,

Copalite, H. J. Bosworth Co., Chicago, Ill.
}

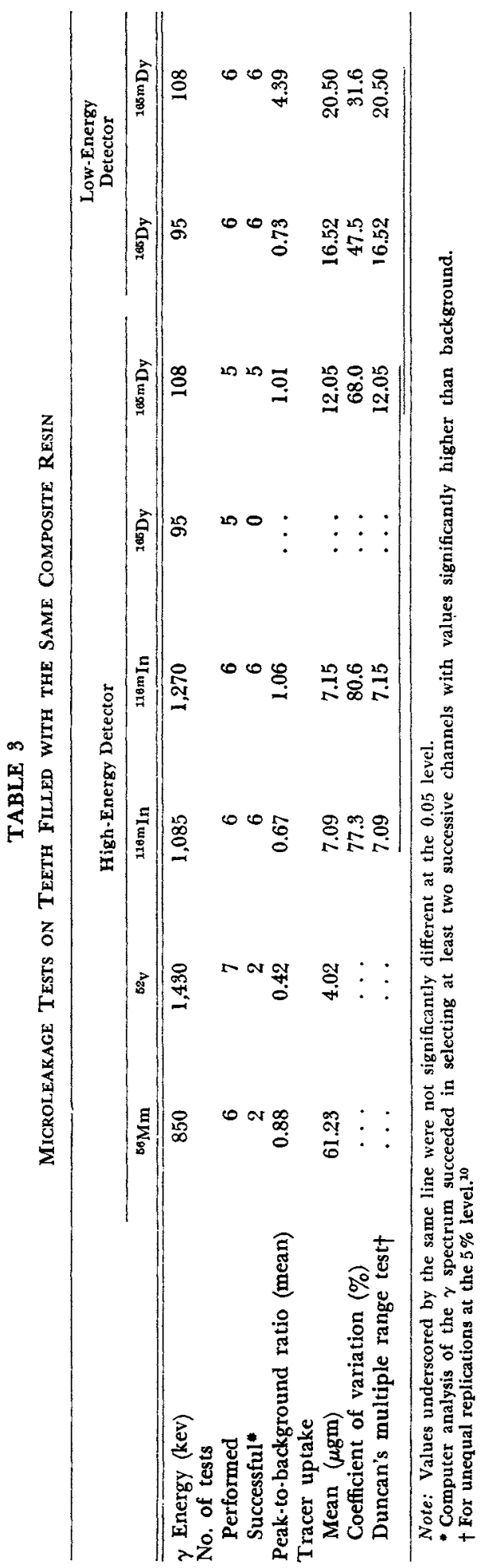


TABLE 4

Comparison of Three Restorative Matertals

\begin{tabular}{|c|c|c|c|}
\hline \multirow[b]{2}{*}{ Conditions } & \multicolumn{3}{|c|}{ Mean Uptake of Dy per Tooth ( $\mu \mathrm{gm})$} \\
\hline & Filled Resin & Unfilled Resin & Silicate Cement \\
\hline Unlined & $20.50(6.47) * \dagger$ & $18.62(2.87) *$ & $13.45(4.79) *+$ \\
\hline Varnish liner & $9.57(7.61)$ & $\cdots$ & $23.61(14.37)$ \\
\hline Temperature-cycled & $100.57(80.87) \dagger$ & . & $104.47(43.06) \ddagger$ \\
\hline
\end{tabular}

of the first and last significant channels of the peak became more sensitive. The number of selected channels for the $108 \mathrm{kev}$ peak increased from a mean of 7.8 channels for the high-energy detector to a mean of 21.3 channels for the low-energy detector; the coefficient of variation for the number of channels selected decreased from 29.2 to $4.8 \%$. Such differences in the mean number of selected channels were highly significant according to Student's $t$ test.

\section{Discussion}

The automatic evaluation procedure is very effective, if careful selection of the best counting conditions is used. The variability of the tracer uptake per tooth, about $30 \%$ in the best example (Table 3 ), demonstrated the influence of the materials being tested rather than that of the testing procedure. Even though the cavities were prepared and filled carefully, many uncertainities were associated with this type of sample. The factors involved in preparation and filling of a cavity cannot remain completely under control and variations in the data should be expected.

The mean tracer uptake and the number of successful tests indicated in Table 3 show clearly that the various tracers behave quite differently in the presence of the same type of marginal leakage. We will not consider Mn because of extremely high background values; the three other tracers seemed to exhibit significantly different penetration properties. Despite the good activation properties of $\mathrm{V}$, it failed to show any repeatable penetration into the marginal gap; five of seven tests did not produce a recognizable $\gamma$ peak. In the case of In, the mean uptake values of the two $\gamma$ peaks were not significantly different, but they were significantly lower than those for Dy and produced considerable scattering of the results. Thus, these two tracers should be considered as less effective than Dy, at least in regard to penetration into the marginal space between a composite resin and the cavity wall. These results answer, in part, the question asked by Going, 2 that is, whether the magnitude of the leakage is completely independent of the tracer.

The data presented in Table 4 suggest the possibility of using this method to compare the marginal leakage around different types of nonmetallic restorations; it does not indicate a definite advantage of one type of material over another, because the study was designed to test the method, not the materials.

Great variations in the mean values indicate a need for more controlled handling of samples in vitro. The technique appears to be sensitive to tracer solution, which can be drawn into cracks and fissures associated with extracted teeth. In the thermal cycling of filled resin restorations, for example, values ranged from $31 \mu \mathrm{gm}$ uptake around more resistant marginal interfaces to $218 \mu \mathrm{gm}$ uptake in teeth that deteriorated more readily under simulated clinical conditions of an extreme nature. It is clear that to overcome some of the problems associated with the nature of the materials, sample sizes should be increased to reduce the influence of the scatter of the data, and therefore, to enhance the power of the test.

A positive regression was found between tracer uptake and the weight of the tooth in the composite resin, whereas no regression could be found for either the unfilled resin or the silicate cement, as shown in Figure 3. Thus, capillary action of the dentin tubules could play a significant role, seemingly proportional to the size of the tooth, if the 


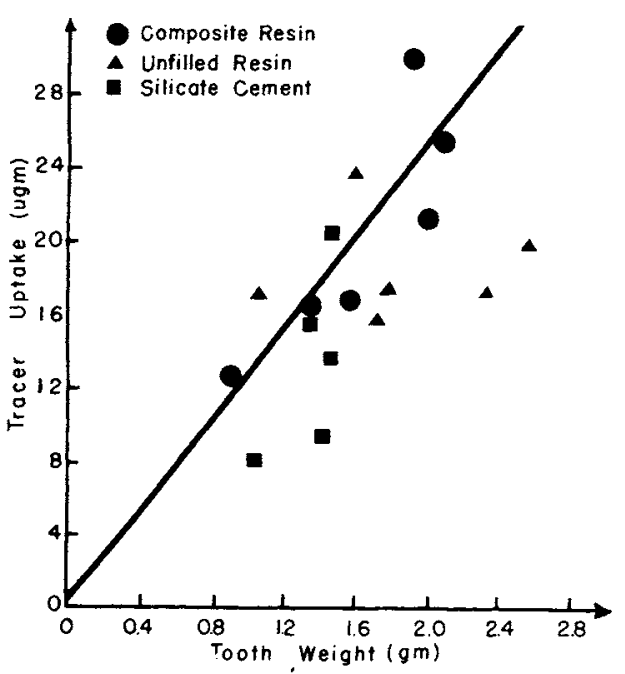

Fig 3.-Tracer uptake as a function of weight of tooth. Only composite filling material exhibited positive regression.

tracer penetrates deeply enough to go beyond the dentinoenamel junction.

Small variations in the weight of the filling material did not show any correlation with tracer uptake. The total counting time exhibited a positive regression with the tracer uptake values and with the tooth weights, as would be expected.

The improved activation method offers the following distinct advantages: The use of Dy provides high selectivity since no interferences were found and would not be normally expected with either tooth or dental restorative materials. Dysprosium also notably shortens the procedure; the activation and decay before counting are very short. With sufficient training, six to ten samples can be run per hour. The computer analysis of the data allows a more thorough evaluation of the $\gamma$ spectrum; this leads to a greater security in the determination of the background and of the significant counts. The weight of the tooth and the filling material were involved in the calculations; this permitted more detailed evaluation of background radiation.

The improved method, with Dy also permits easy analysis of whole teeth after soaking in the tracer solution, whereas the original method proposed by Going, Myers, and
Prussin 1 required sectioning of the tooth samples to minimize background radiation and gain sensitivity. The improved technique also could be modified with specific changes in sample preparation and handling to permit its use in vivo. ${ }^{1}$ It is possible that the sensitivity gained by this method through use of short activation time and counting period could be adapted, with specific precautions and handling procedures, to the evaluation of metallic restorative materials for leakage.

The method has two disadvantages, which result directly from the nature of the proposed tracer. The sensitivity of the method to small defects in natural teeth is enhanced by the high sensitivity of the tracer, and the $\gamma$-ray emission characteristics of Dy (low energy and short half-life) require a low-energy detector and a fast transfer system between the reactor core and the counting room.

\section{Conclusions}

The evaluation of marginal penetration of dental restorations in whole teeth was conducted by use of an in vitro method of neutron activation analysis. Three theoretically selected tracers, V, In, and Dy, were compared with $\mathrm{Mn}$, which had been shown to be unsuccessful because of normal variations in teeth and restorative materials.

Dysprosium was the most successful tracer for the following reasons: (1) high selectivity, which permitted the analysis to be carried out on whole, unsectioned teeth (up to now no Dy has been detected in either teeth or restorative materials) ; (2) rapid activation and counting procedure; (3) high realiability; and (4) high sensitivity, indicated by a high peak-to-background ratio.

Vanadium and In tracers were not as successful because of their poor reliability in the evaluation of the $\gamma$ spectra and the high scatter of results, which occurred partly because of the low peak-to-background ratios.

The use of the $165 \mathrm{~m}$ Dy peak resulted in successful evaluations and the lowest coefficient of variation for microleakage tests on whole teeth containing a composite restoration. A preliminary study of three nonmetallic restorative materials indicated that by carefully controlling specimen preparation statistical differences in leakage between materials and techniques should be discernible with this method. 


\section{References}

1. GoIng, R.E.; MXers, H.N.; and Prussin, S.G.: Quantitative Method for Studying Microleakage In Vivo and In Vitro, $J$ Dent Res 47: 1128-1132, 1968.

2. GoING, R.E.: Microleakage Around Dental Restorations: A Summarizing Review, JADA 84: 1349-1357, 1972.

3. Dennison, J.B.: An Evaluation of Finishing Procedures and Microleakage on Restorative Composite Resins, MS thesis, University of Michigan, School of Dentistry, Ann Arbor, Mich, 1970.

4. Handbook of Chemistry and Physics, 44th ed, Cleveland; Chemical Rubber Publishing Co., 1963, pp 450-515.

5. Duivenstijn, A.J., and Venverloo, L.A.J.: Spectrométrie gamma en pratique, Paris: Editions Dunod, 1965, pp 125-141.
6. Perdijon, J.: L'analyse par activation, Paris: Editions Masson, 1967, p 7.

7. Currie, L.A.: Limits for Qualitative Detection and Quantitative Determination. Application to Radiochemistry, Anal Chem, 40: 586-593, 1968.

8. Willard, H.H., and DieHL, H.: Advanced Quantitative Analysis, New York: Van Nostrand, 1943, p 238.

9. Haerdi, W.; Martin, E.; and Monnier, D.: Analyse Radiochimique par Activation aux Neutrons Thermiques. Dosage direct $\mathrm{du}$ vanadium dans un acier 18/8, par mesure de l'activité du vanadium 52, Helv Chim Acta 46: 1573-1580, 1963.

10. Steel, R.G.D., and Torrie, J.H.: Principles and Procedures of Statistics, New York: McGraw-Hill, 1960, p 114. 